\title{
Event-by-Event Estimate of Muon Energy Loss in ATLAS
}

\author{
Konstantinos Nikolopoulos, Dimitrios Fassouliotis, Christine Kourkoumelis, and Alan Poppleton
}

\begin{abstract}
The ATLAS Muon Spectrometer is designed to provide efficient and precise stand-alone momentum measurement for muons of momentum up to $\mathrm{O}(1 \mathrm{TeV} / \mathrm{c})$. The fluctuations of the muon energy loss in the material upstream of the Muon Spectrometer could deteriorate the physics performance, which requires the momentum to be determined at the interaction point. A method to account for this energy loss using the calorimeter information is presented. The method provides an estimate of the energy loss on an event-by-event basis thus reducing the statistical fluctuations. The improvement in the momentum resolution by the significant suppression of the non-Gaussian tails of the energy loss is demonstrated using simulated single muon data. The performance of the method on physics samples with muons in the final state is also briefly discussed.
\end{abstract}

Index Terms-ATLAS, energy loss, LHC, muon reconstruction.

\section{INTRODUCTION}

A TLAS [1], currently under installation, will exploit the full physics potential of the LHC [2]. The ATLAS Muon Spectrometer (MS) [3] is designed to perform efficient and accurate stand-alone muon identification and momentum measurement (Stand-Alone muon reconstruction) [4] more than a hundred radiation lengths from the interaction point. Thus, the back-tracking of a muon to the production vertex should appropriately take into account the material induced effects, namely the energy loss and the multiple scattering. To achieve optimum resolution, the MS and the Inner Detector (ID) [5] measurements must be combined (ComBined muon reconstruction) [6]. As shown in Fig. 1, in the low momentum regime, the precision of the momentum measurement is determined by the accuracy of the ID, while the MS is used mainly to provide the muon identification. At higher momenta, the MS provides most of the information in the momentum measurement. The crossover point varies between $P_{T}=80 \mathrm{GeV} / \mathrm{c}$ in the barrel region $(|\eta|<1)$ and $P_{T}=20 \mathrm{GeV} / \mathrm{c}$ in the forward region.

The energy loss of muons follows roughly a Landau distribution ([7], [8]), which is asymmetric. In order to determine the muon momentum at the interaction point, the muon energy loss upstream of the MS should be added to the momentum determined by the stand-alone MS measurement. More precisely, the energy loss estimate is treated as an additional parameter in the track fitting procedure, which is given an estimated value and an uncertainty according to the energy loss estimation method used.

Manuscript received January 9, 2007; revised June 12, 2007. This work was supported by the European Social Fund and National Resources-(EPEAEK II) PYTHAGORAS II.

K. Nikolopoulos, D. Fassouliotis, and C. Kourkoumelis are with the Physics Department, University of Athens, GR-15771 Athens, Greece.

A. Poppleton is with CERN, CH-1211 Geneva, Switzerland.

Digital Object Identifier 10.1109/TNS.2007.905157

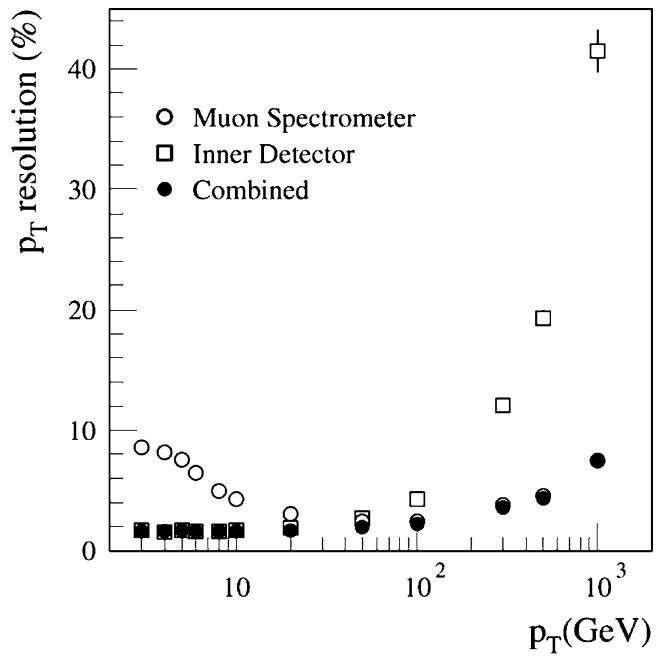

Fig. 1. The resolution of the ATLAS detector on the muon momentum measurement from the Muon Spectrometer, the Inner Detector and their combination, averaged over pseudorapidity, as a function of $P_{T}$. Reprinted from "ATLAS Detector and Physics Performance Technical Design Report," CERN-LHCC-99-14/15 (1999), with permission.

Traditional energy loss corrections use a parametrization of either the most probable (mop) or the mean energy loss as a function of the particle's momentum and the amount of material it traversed. This statistical approach, although reasonable in several cases, cannot account for the event-by-event fluctuations of the energy loss. These fluctuations contribute to a significant non-Gaussian tail of the energy loss resolution, which propagates to the momentum resolution at the interaction point. A method accounting for the energy loss fluctuations on an event-by-event basis using the calorimeter information is appropriate and its development is presented. Furthermore, it has been found that an improved energy loss resolution is achieved by combining the calorimeter measurement with the mop parametrization in the so-called "hybrid" method. A comparison between the results of the hybrid method and parametrization alone is also presented.

Although the measurement of ionization energy loss has been extensively used for high energy particle identification since the early 1950s [9], this has not been the case for muon momentum reconstruction. An overview of the early energy loss measurements and calculations is given in [10], while more recent reviews can be found in [11] and [12]. In the pre-LHC energy regime, the muon could be considered as a minimum ionizing particle, and furthermore the common practice was to perform the particle tracking as close to the production vertex as possible in order to minimize the detector material effects. However, in [13] the use of calorimetry to measure the electromagnetic 


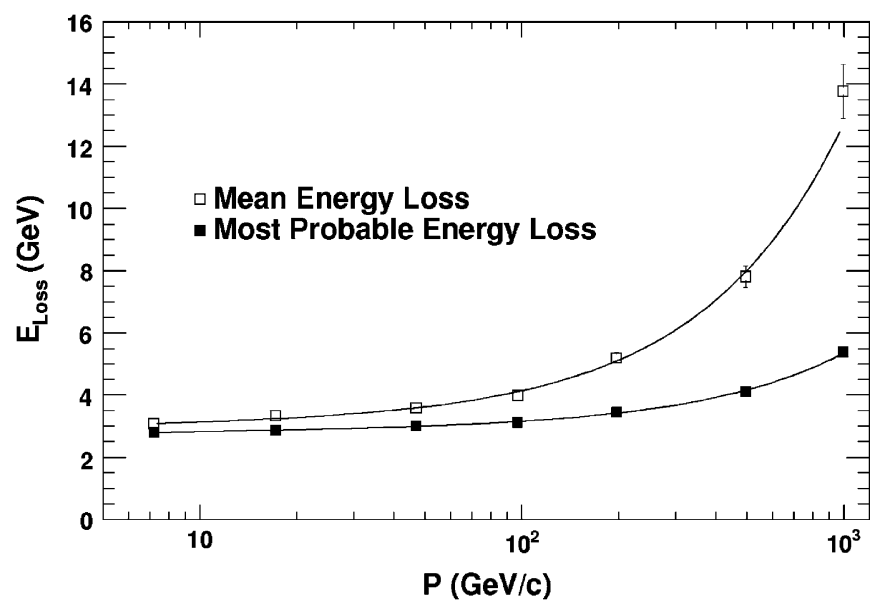

Fig. 2. Most probable and mean energy loss as a function of muon momentum for $0.4<|\eta|<0.5$.

showers induced by $\mathrm{TeV}$ muons in future experiments is suggested, while in [14] the response of the ATLAS tile calorimeter prototype to muons is studied in detail, and its possible use for the muon energy loss determination is considered.

For this study, all samples were fully simulated using the GEANT 4 [15] based ATLAS detector simulation [16], without pile-up. The results of preliminary studies with pile-up are encouraging. The physics samples were generated with PYTHIA [17].

\section{PARAmetrization OF THE MuON ENERGY LOSS IN ATLAS}

The statistical properties of the energy loss depend only on the momentum and the traversed material. The mop energy loss is parametrized by a continuous three parameter function of momentum $(P)$ expressing the physical aspects of the energy loss process:

$$
E_{\mathrm{Loss}}^{\mathrm{Par}}=a_{0}+a_{1} \cdot \ln \left(A \cdot P^{2}\right)+a_{2} \cdot P
$$

The first term of (1) describes the minimum ionizing part of the muon energy loss, while the second and the third terms describe the relativistic rise and the radiative effects, respectively. The parameters themselves (except for parameter A) are functions of pseudorapidity $(\eta)$, accounting for the different material traversed in a given direction, and their values were determined using single muon samples of constant momentum. The aforementioned function can be used for the parametrization of the mean energy loss as well. In Fig. 2, the mop and mean energy loss are presented as a function of $P$ for a particular $\eta$ bin together with their parametrized description. Additionally, the width of the energy loss distribution, $\sigma_{\text {Landau }}$, was parametrized as a function of momentum (and $\eta$ ) by a first degree polynomial, while the uncertainty of the mop energy loss estimate was determined by measuring the $90 \%$ probability intervals in each side of the energy loss distribution (asymmetric uncertainty) and transforming them to Gaussian standard deviations.

The parametrizations were validated with independent single muon samples of constant $P_{T}$ in the range $10-1000 \mathrm{GeV} / \mathrm{c}$.

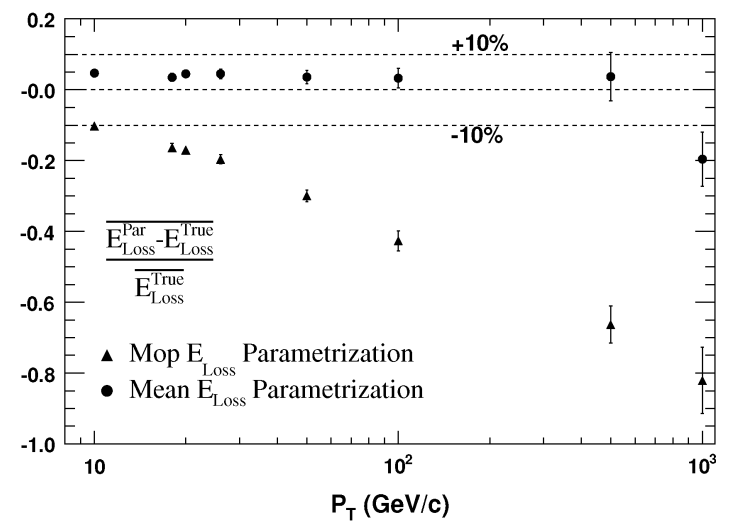

(a)

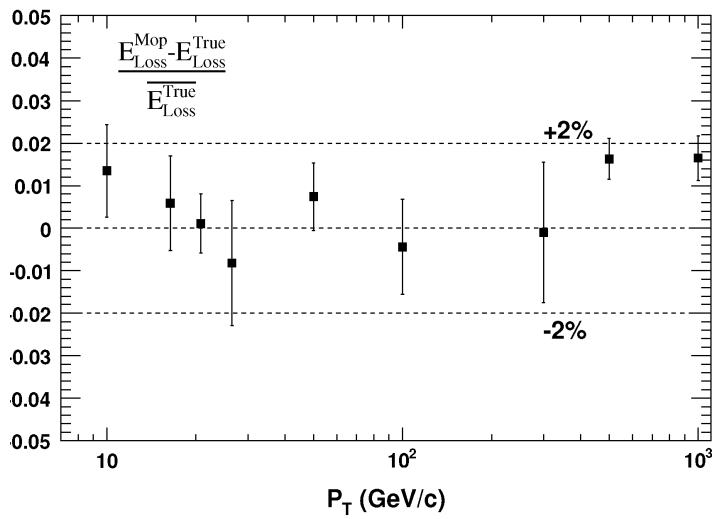

(b)

Fig. 3. Performance of the parametrizations described in the text as a function of $P_{T}$. (a) The mean of the distribution $E_{\mathrm{L} \text { ass }}^{\text {Par }}-E_{\mathrm{L} o s s}^{\text {True }}$ normalized to the mean $E_{\text {Loss }}^{\text {True }}$. (b) The Gaussian mean for the distribution $E_{\text {Loss }}^{\text {Mop }}-E_{\text {Loss }}^{\text {True normalized }}$ to the mean $E_{\text {Loss }}^{\text {True }}$.

Their performance in terms of the difference between the parametrized and the true energy loss, normalized to the mean true energy loss, is summarized in Fig. 3. A distinction is made between the mean value, Fig. 3(a), and the Gaussian mean, Fig. 3(b), of this distribution. In the first case, the mean parametrization provides a satisfactory description at the expense of overestimating the energy loss of muons at the core of the asymmetric Landau distribution. In the second case, the mop parametrization, which underestimates the mean energy loss, is well centered about the core of the energy loss distribution.

As shown in Section IV, the calorimeter information is used to determine the large energy losses. Thus, the mop parametrization is more appropriate to describe the muons with energy loss in the core of the distribution. In the case when no calorimeter information is available, the overall momentum distribution is a convolution of the asymmetric Landau energy loss and the Gaussian MS resolution. A correction using an appropriate truncated mean is applied to account for the effect of this convolution.

\section{Description of the MeAsurement of THE MUON ENERGY LOSS IN ATLAS}

Each muon track is extrapolated back to the ATLAS Calorimeters [18] where measurement cones are formed in the $(\eta, \phi)$ plane (small square of Fig. 4). The cone radii are chosen 


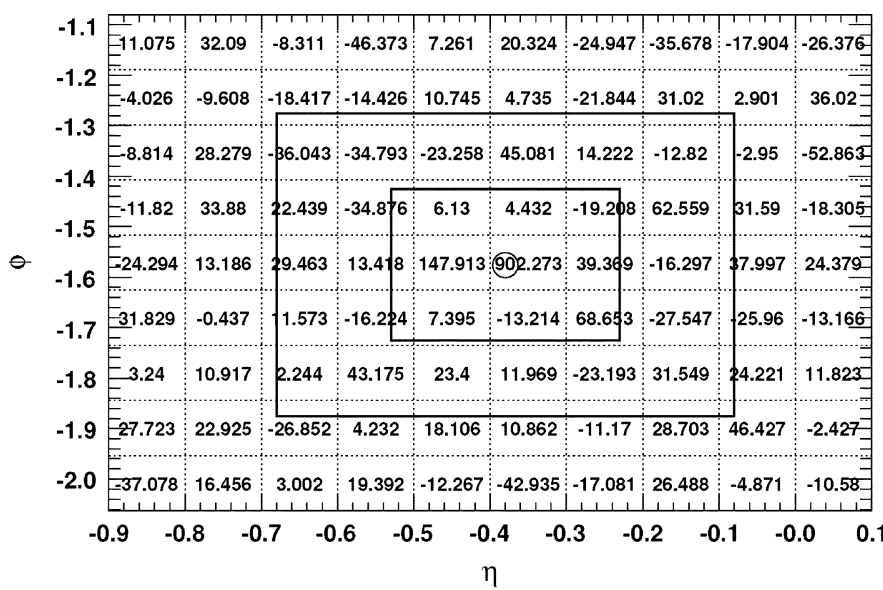

Fig. 4. Tile calorimeter sampling expanded in the $(\eta, \phi)$ plane to illustrate the chosen measurement and isolation areas (small and large square, respectively) with respect to the cell size. The energy recorded in each cell (in $\mathrm{MeV}$ ) is shown for a typical isolated muon (small circle).

separately for each calorimeter according to its segmentation and the extrapolation error: $(\delta \eta, \delta \phi)=0.15$ for the hadronic calorimeters and $(\delta \eta, \delta \phi)=0.075$ for the electromagnetic calorimeter. Within each cone, the recorded energy of the cells is summed, using a threshold equal to 4 times the RMS noise. In the presence of pile-up, it is foreseen to subtract the mean energy contribution. In the case where no significant energy above noise is observed in a calorimeter segment, the most probable energy deposition in this segment is used.

The electromagnetic and hadronic parts are summed and corrected for the e/mip ratio ([14], [19], [20]) and for the energy deposited in the additional passive material traversed by the muon (e.g., the cryostats). The former correction is necessary because the ATLAS calorimeters are calibrated in the electromagnetic energy scale, while the latter correction is determined using the material map of the GEANT 4 simulation of the ATLAS detector and the mop parametrization derived in the previous section. This passive material represents on average $10 \%$ of the total material upstream of the MS, but can reach as much as $20 \%$ at some $\eta$ regions. The resulting energy is the measured estimate of the muon energy loss upstream of the MS.

\section{DESCRIPTION OF THE HYBRID}

\section{MEASUREMENT/PARAMETRIZATION METHOD}

The muon energy loss, being Landau distributed, is characterized by a core region around the mop value, plus a long tail with large energy loss. The calorimetric measurement of this tail is performed with a relative resolution which scales as $E^{-1 / 2}$, providing an accurate description of the large energy losses. On the other hand, the mop parametrization offers high accuracy for muons with energy loss in the core of the Landau distribution. Thus, an improved energy loss resolution is achieved when these two approaches are combined (hybrid method): the calorimetric energy loss measurement is used when the energy deposition is significantly larger than the mop value; otherwise the mop parametrization is used. The transition value between the two approaches is $E_{\text {mop }}+2 \cdot \sigma_{\text {Landau }}$, which was found to provide an unbiased momentum reconstruction despite the asymmetry of the Landau distribution. The fraction of muons which make
TABLE I

SEQUENCE OF CUTS FOR THE ISOLATION TAGGING OF MUONS

\begin{tabular}{|c|c|c|}
\hline & $\begin{array}{l}\text { Muons From } \\
\text { W Decays } \\
(\%)\end{array}$ & $\begin{array}{c}\text { Muons From } \\
\text { Quark Decays } \\
(\%)\end{array}$ \\
\hline No Cut & 100.00 & 100.00 \\
\hline Calorimeter Isolation Cone & 91.99 & 22.52 \\
\hline$P_{T}>15 \mathrm{GeV} / \mathrm{c}$ & 82.87 & 4.45 \\
\hline ID Track Isolation & 80.45 & 0.964 \\
\hline \multicolumn{3}{|c|}{ Accuracy for the Muons Tagged as Isolated } \\
\hline$\left|E_{\text {Loss }}^{E s t i m}-E_{\text {Loss }}^{T r u e}\right|<6 \mathrm{GeV}$ & 78.55 & 0.815 \\
\hline$\left|E_{\text {Loss }}^{\text {Estim }}-E_{\text {Loss }}^{\text {True }}\right|>6 \mathrm{GeV}$ & 0.64 & 0.148 \\
\hline
\end{tabular}

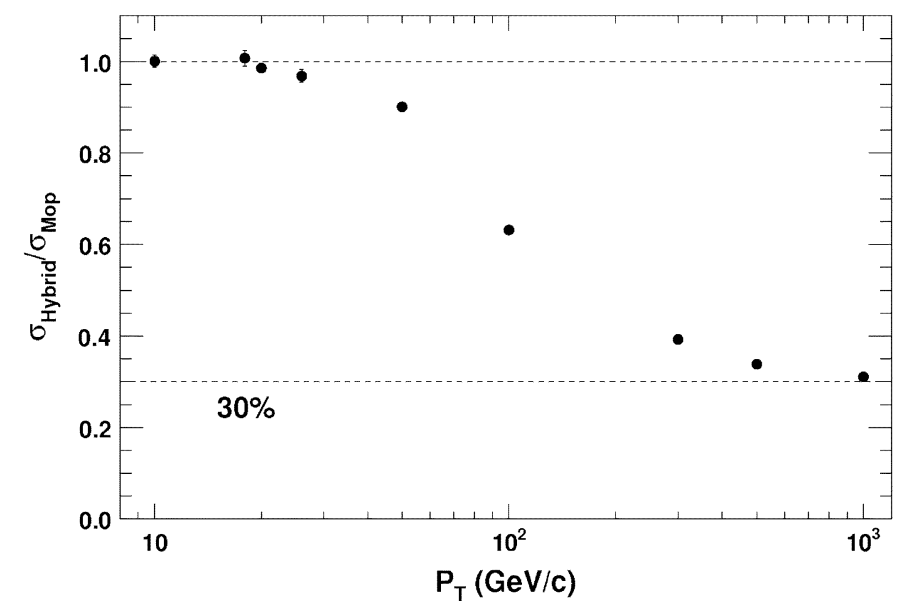

Fig. 5. The ratio of the energy loss resolution for the hybrid method with respect to the mop parametrization in single muon events as a function of $P_{T}$.

use of the calorimeter measurement increases with increasing momentum and reaches $60 \%$ at $P_{T}=1 \mathrm{TeV} / \mathrm{c}$.

The calorimetric energy loss measurement is reliable only for isolated muons. A study of muon isolation has been performed using calorimeter and charged track isolation and muon $P_{T}$ criteria. Calorimeter isolation is based on the energy deposition inside an isolation cone as shown in Fig. 4, while charged track isolation is based on the number of ID tracks accompanying the muon at the interaction point. The evaluation of the method was performed using a fully simulated $t \bar{t}$ sample, where the $\mathrm{W}$ bosons were forced to decay to muon plus neutrino. Muons produced by the semi-leptonic decays of $\mathrm{b}$ or $\mathrm{c}$ quarks tend to be non-isolated, while those from $\mathrm{W}$ decays tend to be isolated. Table I shows the sequence of the requirements and the cumulative efficiencies. The hybrid method is only used when the muon is tagged as isolated; otherwise the energy loss is determined by the mop parametrization.

\section{Performance With Single Muon Events (STAND-ALONE AND COMBINED)}

The relative performance of the two different methods, namely the mop parametrization (Section II) and the hybrid method (Section IV), for the muon energy loss estimation is compared using fully simulated single muon samples of constant $P_{T}$ in the range between $10 \mathrm{GeV} / \mathrm{c}$ and $1000 \mathrm{GeV} / \mathrm{c}$.

Fig. 5 shows the ratio of the energy loss resolution for the hybrid method, $\sigma_{\text {Hybrid }}$, with respect to the mop parametrization method, $\sigma_{\mathrm{Mop}}$. For low $P_{T}$ values, the ratio is close to 1 , because in this region the two methods are roughly equivalent. However, 


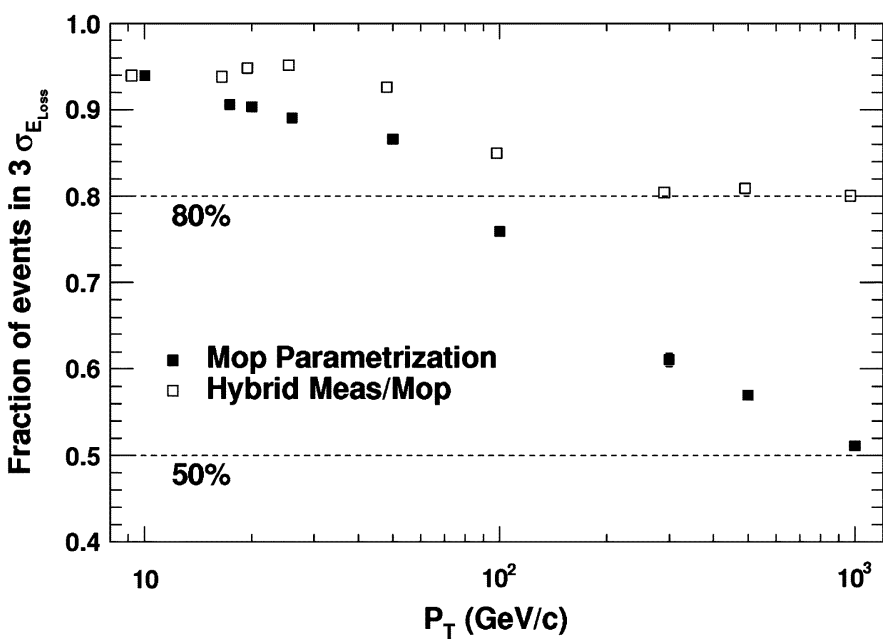

Fig. 6. The fraction of single muon events within a fixed window of the energy loss resolution, $\pm 3 \cdot \sigma_{\text {Hybrid }}$, for the hybrid and the mop parametrization method as a function of $P_{T}$.

the ratio then decreases with increasing $P_{T}$, approaching $30 \%$ at $P_{T}=1 \mathrm{TeV} / \mathrm{c}$. Thus, a significant improvement in the energy loss estimate is gained by the use of the hybrid method. In Fig. 6, the fraction of events within a fixed window of the energy loss resolution, $\pm 3 \cdot \sigma_{\text {Hybrid }}$, is shown for both methods as a function of $P_{T}$. The reduction of the tails when using the hybrid method is significant for $P_{T}>25 \mathrm{GeV}$ and reaches $60 \%$ for $P_{T}=1 \mathrm{TeV} / \mathrm{c}$.

The improvement made to the momentum resolution at the interaction point is presented in Fig. 7, where the ratio of the resolution obtained with the hybrid method with respect to the mop parametrization is shown as a function of $P_{T}$. For the stand-alone muon reconstruction, Fig. 7(a), an improvement of 5-10\% is observed over most of the momentum region. At low $P_{T}$, the influence of the energy loss fluctuations in the momentum resolution is more significant, but the benefit from the hybrid method is moderate because for most muons the energy loss is consistent with the mopexpectation and due to the $P_{T}$ cut imposed in the isolation criteria. At high $P_{T}$, the benefit from the hybrid method is larger, but so is the momentum uncertainty originating from the intrinsic MS resolution. The latter reduces the impact of the energy loss fluctuations on the momentum resolution. A significant part of the improvement achieved using the hybrid method is maintained with the combined muon reconstruction as shown in Fig. 7(b).

\section{Performance on Mass Reconstruction of RESONANCES DECAYING TO MuONS}

The improvement in the muon momentum resolution leads to a corresponding improvement in the mass resolution of resonances decaying to muons, these quantities are connected by (2).

$$
\frac{\delta M}{M} \approx \frac{1}{\sqrt{2}} \frac{\delta P}{P}
$$

In Table II, the resolution of the methods on dimuon invariant mass reconstruction is presented for the Standard Model electroweak boson $Z$ and for a hypothetical heavy vector boson $Z^{\prime}$. Stand-alone and combined muon reconstruction are considered. For the $Z$, the improvement follows the properties presented above for single muons, both in the stand-alone and the combined reconstruction case. Some of the improvement in the

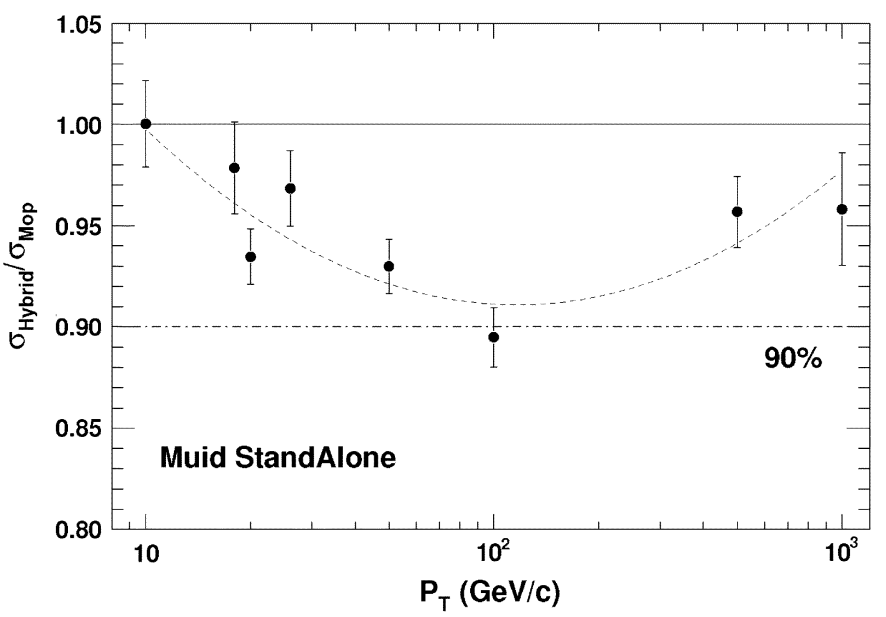

(a)

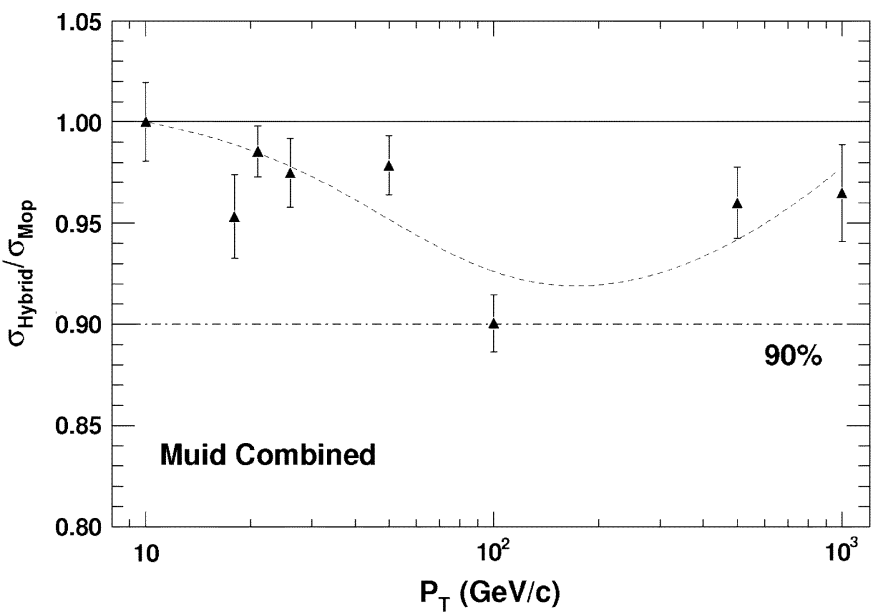

(b)

Fig. 7. Ratio of the single muon momentum resolution for the hybrid method with respect to the mop parametrization as a function of $P_{T}$. (a) Stand-alone reconstruction: A parabolic curve is fitted since unity is expected in the low and high $P_{T}$ extremes. (b) Combined reconstruction: The above fitted curve is weighted to allow for the relative contribution of the stand-alone measurement to the combined muon reconstruction, as seen in Fig. 1.

TABLE II

DiMUON MASS RESOLUTION

\begin{tabular}{|c|c|c|c|}
\hline Process & $\begin{array}{c}\text { Parametrization } \\
(\%)\end{array}$ & $\begin{array}{c}\text { Hybrid } \\
(\%)\end{array}$ & $\begin{array}{c}\text { Improvement } \\
(\%)\end{array}$ \\
\hline \hline \multicolumn{4}{|c|}{ Stand-alone Reconstruction } \\
\hline$Z \rightarrow \mu^{+} \mu^{-}$ & $2.84 \pm 0.05$ & $2.65 \pm 0.04$ & $7 \pm 2$ \\
\hline $\begin{array}{c}Z^{\prime} \rightarrow \mu^{+} \mu^{-} \\
\left(1 \mathrm{TeV} / \mathrm{c}^{2}\right)\end{array}$ & $4.69 \pm 0.09$ & $4.27 \pm 0.08$ & $9 \pm 2$ \\
\hline \hline \multicolumn{4}{|c|}{ Combined Reconstruction } \\
\hline$Z \rightarrow \mu^{+} \mu^{-}$ & $1.59 \pm 0.03$ & $1.54 \pm 0.02$ & $3 \pm 1$ \\
\hline $\begin{array}{c}Z^{\prime} \rightarrow \mu^{+} \mu^{-} \\
\left(1 \mathrm{TeV} / \mathrm{c}^{2}\right)\end{array}$ & $4.38 \pm 0.08$ & $4.04 \pm 0.07$ & $8 \pm 2$ \\
\hline
\end{tabular}

$Z^{\prime}$ sample is due to the fact that the hybrid method accounts for the final state radiation, while the parametrization does not. Stand-alone reconstruction is almost as good as combined reconstruction for $Z^{\prime}$ because at high momenta the momentum resolution is dominated by the MS measurement.

The resolutions on the $Z$ mass from the hybrid and the mop parametrization methods are directly compared in Fig. 8(a); the corresponding comparison for the $Z^{\prime}$ mass is presented in Fig. 8(b). Quantitively, for stand-alone reconstruction, the 


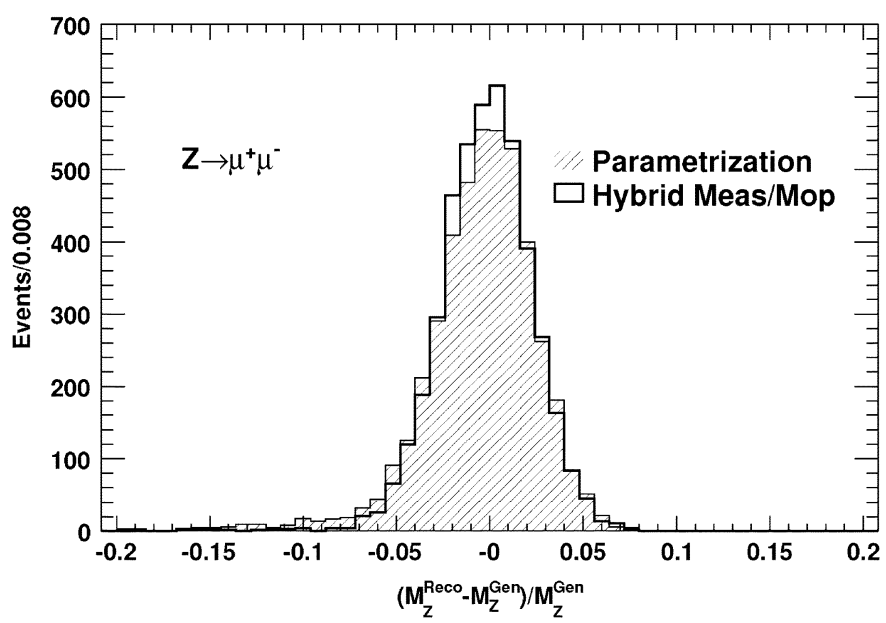

(a)

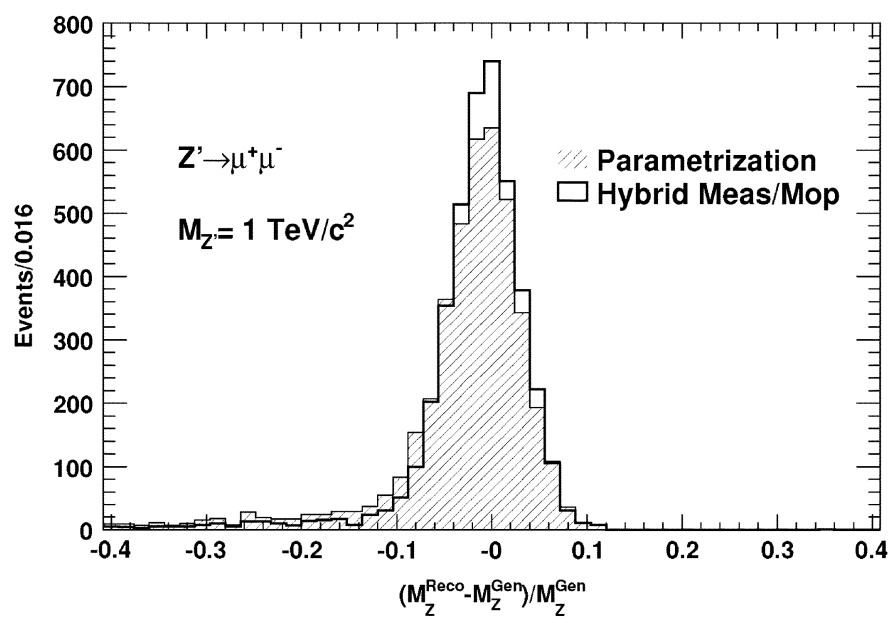

(b)

Fig. 8. The resolution on (a) the $Z$ and (b) the $Z^{\prime}$ mass stand-alone reconstruction for the hybrid (empty histogram) and the mop parametrization (hatched histogram) methods. The tails of the resolution are due to muon energy losses on the Landau tail. Significant increase of the signal in the peak region is observed when using the hybrid instead of the parametrization method.

number of $Z$ events inside a fixed window, $\pm 2 \cdot \sigma_{\text {Hybrid }}$ of the mass resolution, is increased by $4 \%$ using the hybrid method. The respective increase for $Z^{\prime}$ events is $7 \%$ for stand-alone and $6 \%$ for combined reconstruction. Therefore, the use of the hybrid method provides a better resolution and consequently a better signal-over-background ratio $(S / B)$, which may improve the statistical significance of a potential discovery.

\section{CONCLUSION}

A method exploiting the calorimeter information to provide accurate estimate of the muon energy loss upstream of the ATLAS Muon Spectrometer is presented. The calorimeter measurement is combined with the most probable energy loss parametrization to obtain an improved resolution. Care is taken to distinguish between isolated and non-isolated muons.

The method significantly improves the resolution on the muon energy loss and decreases the non-Gaussian tails with respect to the simple parametrization method. The improvement on the energy loss resolution propagates to the stand-alone muon reconstruction providing on average a $5 \%$ improvement on the muon stand-alone momentum resolution in the $P_{T}$ range from $10 \mathrm{GeV} / \mathrm{c}$ to $1 \mathrm{TeV} / \mathrm{c}$, reaching $10 \%$ for $P_{T}=100 \mathrm{GeV} / \mathrm{c}$. A significant part of the improvement on the stand-alone muon reconstruction is inherited by the combined muon reconstruction. Moreover, the improvement on the stand-alone muon reconstruction facilitates the matching between the MS and the ID tracks resulting to a more efficient combined reconstruction.

The improvement on the momentum resolution has an impact on the reconstruction of resonances decaying to muons $\left(Z, Z^{\prime}\right)$, thus improving the signal-over-background ratio $(S / B)$ and the physics discovery potential.

\section{ACKNOWLEDGMENT}

The authors would like to thank S. Willocq, D. Levin, and K. Cranmer for fruitful discussions. Suggestions and comments on the manuscript by R. Clifft should also be acknowledged. This work has been performed within the ATLAS collaboration making use of tools which are the result of collaboration-wide efforts.

\section{REFERENCES}

[1] ATLAS Collaboration, "ATLAS Detector and Physics Performance Technical Design Report,” CERN-LHCC-99-14/15, 1999.

[2] O. Bruning, P. Collier, P. Lebrun, S. Myers, R. Ostojic, J. Poole, and P. Proudlock, "LHC Design Report Vol I: The LHC Main Ring," CERN2004-003-V-1, 2004.

[3] ATLAS Collaboration, "ATLAS Muon Spectrometer Technical Design Report," CERN-LHCC-97-22, 1997.

[4] D. Adams et al., "Track Reconstruction in the ATLAS Muon Spectrometer with Moore," ATLAS Internal Note, ATL-SOFT-2003-007, 2003.

[5] ATLAS Collaboration, "ATLAS Inner Detector Technical Design Report Vol I," CERN-LHCC-97-16, 1997.

[6] T. Lagouri et al., "A muon identification and combined reconstruction procedure for the ATLAS detector at the LHC at CERN," IEEE Trans. Nucl. Sci., vol. 51, no. 6, pt. 1, pp. 3030-3033, Dec. 2004.

[7] L. D. Landau, "On the energy loss of fast particles by ionization," $J$. Phys. (USSR), vol. 8, pp. 201-, 1944.

[8] P. V. Vavilov, "Ionization losses of high-energy heavy particles," Sov. Phys. JETP., vol. 5, p. 749, 1957.

[9] A. I. Alikhanov, V. A. Lubimov, and G. P. Eliseiev, "High precision measurement of the ionizing power of fast charged particles with the help of multi-layer proportional counters," in Proc. CERN Symp. High Energy Accelerators Pion Phys., 1956, vol. 2, p. 87.

[10] W. W. M. Allison and J. H. Cobb, "Relativistic charged particle identification by energy loss," Ann. Rev. Nucl. Part. Sci., vol. 30, p. 253, 1980.

[11] M. Hauschild, "Progress in $\mathrm{dE} / \mathrm{dx}$ techniques used for particle identification," Nucl. Instrum. Meth. A., vol. 379, p. 436, 1996.

[12] J. Va'vra, "Particle identification methods in high energy physics," Nucl. Instrum. Meth. A., vol. 453, p. 262, 2000.

[13] U. Becker et al., "Precision muon detectors in the TeV region," Nucl. Instrum. Meth. A., vol. 253, p. 15, 1986.

[14] Z. Ajaltouni et al., "Response of the ATLAS tile calorimeter prototype to muons," Nucl. Instrum. Meth. A., vol. 388, p. 64, 1997.

[15] S. Agostinelli et al., "GEANT4: A simulation toolkit," Nucl. Instrum. Meth. A., vol. 506, p. 250, 2003, [GEANT4 Collaboration].

[16] A. Rimoldi et al., "ATLAS detector simulation: Status and outlook," in Proc. 9th ICATPP Conf. Astroparticle, Particle, Space Phys., Detectors Med. Phys. Applicat., Villa Erba, Como, Italy, Oct. 2005, pp. 17-21.

[17] T. Sjostrand, S. Mrenna, and P. Skands, "PYTHIA 6.4 physics and manual," JHEP, vol. 0605, p. 026, 2006.

[18] ATLAS Collaboration, "ATLAS Calorimeter Performance," CERNLHCC-96-40, 1996.

[19] B. Dowler et al., "Performance of the ATLAS hadronic end-cap calorimeter in beam tests," Nucl. Instrum. Meth. A., vol. 482, p. 94, 2002, [ATLAS Liquid Argon HEC Collaboration].

[20] B. Aubert et al., "Performance of the ATLAS electromagnetic calorimeter barrel module 0," Nucl. Instrum. Meth. A., vol. 500, p. 202, 2003, [Erratum-ibid. A. 517 (2004) 399] [ATLAS Electromagnetic Liquid Argon Calorimeter Group]. 